OPEN

SUBJECT AREAS:

STATISTICAL PHYSICS, THERMODYNAMICS AND NONLINEAR DYNAMICS

COMPUTATIONAL NEUROSCIENCE

Received

24 December 2013

Accepted

19 February 2014

Published

13 March 2014

Correspondence and requests for materials should be addressed to L.d.A. (lucilla. dearcangelis@unina2.

\title{
Brain modularity controls the critical behavior of spontaneous activity
}

\author{
R. Russo' ${ }^{1}$ H. J. Herrmann ${ }^{2,3}$ \& L. de Arcangelis ${ }^{4}$
}

\begin{abstract}
'Physics Department, University of Naples Federico Il, Napoli, Italy, ${ }^{2}$ Institute Computational Physics for Engineering Materials, ETH, Zürich, CH, ${ }^{3}$ Departamento de Física, Universidade Federal do Ceará, 6045 1-970 Fortaleza, Ceará, Brazil, ${ }^{4}$ Department of Industrial and Information Engineering, Second University of Naples and INFN Gr. Coll. Salerno, Aversa (CE), Italy.
\end{abstract}

The human brain exhibits a complex structure made of scale-free highly connected modules loosely interconnected by weaker links to form a small-world network. These features appear in healthy patients whereas neurological diseases often modify this structure. An important open question concerns the role of brain modularity in sustaining the critical behaviour of spontaneous activity. Here we analyse the neuronal activity of a model, successful in reproducing on non-modular networks the scaling behaviour observed in experimental data, on a modular network implementing the main statistical features measured in human brain. We show that on a modular network, regardless the strength of the synaptic connections or the modular size and number, activity is never fully scale-free. Neuronal avalanches can invade different modules which results in an activity depression, hindering further avalanche propagation. Critical behaviour is solely recovered if inter-module connections are added, modifying the modular into a more random structure.

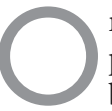

ne of the crucial questions in biology is the relation between structure and function: To what extent the particular structure of a living system controls the performance in specific functions, or is pathological behaviour related to deviations from an optimal structure? This question is of remarkable interest for what concerns the human brain. Modularity is the main feature of the brain, composed of functional areas, whose existence is well known since more than a century. The brain functional connectivity network has received wide attention in the literature in recent years. In particular the functional network has been found to exhibit scale free properties ${ }^{1}$, i.e., the absence of a typical connectivity degree, and small world features ${ }^{2}$. Experimental results indicate that healthy brains exhibit small world features, whereas patients affected by neurological diseases are characterized by different networks. In particular, schizophrenic patients ${ }^{3}$ or brain tumor patients ${ }^{4}$ exhibit a more random architecture of the underlying network. Within this context, recently Gallos et $\mathrm{al}^{5}$ have developed a detailed analysis of fMRI data evidencing the complexity of the modular structure of human brain: The functional network is composed of a set of hierarchically organized modules made of strong links. These modules are selfsimilar structures, not small-world. However, modules are connected by weaker ties, which make the network small world, preserving the well-defined modules. Remarkably, weak ties are strategically organized in order to maximize information transfer with minimal wiring $\operatorname{cost}^{6-8}$.

Neuronal activity is the fundamental process leading to the complex brain functions. Even in absence of external stimuli, the brain undergoes a spontaneous activity which represents about $30 \%$ of the overall activity. Spontaneous neuronal activity consists of bursts of firing neurons that can last from a few to several hundreds of milliseconds and, if analysed at a finer temporal scale, exhibit a complex structure in terms of neuronal avalanches. Indeed, in vitro experiments have recorded avalanche activity ${ }^{9,10}$ from mature cultures of rat cortex, whose size and duration distributions follow a power law with exponents, $\sim-1.5$ and $\sim-2$, respectively. This is a typical feature of a system acting in a critical state, where large fluctuations are present and the response does not have a characteristic size. The same critical behavior has been measured in vivo from rat cortical layers ${ }^{11}$, from the cortex of awake adult rhesus monkeys ${ }^{12}$, as well as for dissociated neurons from rat hippocampus ${ }^{13,14}$ or leech ganglia $^{13}$. All these experiments record electro-physiological activity by means of microelectrode arrays, sampling small areas of the cerebral system. Criticality in brain activity has been also investigated at a larger scale. In particular, from fMRI recordings of spontaneous activity in healthy subjects ${ }^{15}$ the activity correlation length scales with the functional area size. Spontaneous brain activity has been also measured in healthy subjects by magnetoencephalography $(\mathrm{MEG})^{16}$ and found to scale with the same exponent of neuronal avalanches. Experimental data do not exhibit cascades involving the entire brain, as expected in a system acting in a critical state. 
The scaling exponents of neuronal avalanches measured in different experiments are quite robust which has stimulated a number of theoretical studies ${ }^{17,18}$ and neuronal models have successfully reproduced the exponents found for neuronal avalanches ${ }^{19-22}$. Several complex networks have been implemented, as well as hierarchical modular networks ${ }^{23}$. However, an important open issue is to verify the critical behavior of spontaneous activity on networks reproducing closely the human brain at a larger scale, as done recently for the role of cortical columns in neural computation ${ }^{24}$. This study addresses the question how the complex human brain structure, and in particular modularity (Fig. 1), affects spontaneous activity. The problem is of great relevance since spontaneous activity plays an important role in evoked activity, i.e., the response of the system to external stimulations.

\section{Results}

We implement a neuronal network model ${ }^{19,25-27}$, able to reproduce the scaling properties of neuronal avalanches on non-modular complex networks, on a structure that has the main statistical properties of the functional network measured in human brains ${ }^{5}$ (see Methods). The aim is to enlighten the dependence of the critical features of neuronal activity on the number of modules $M$, their size $N_{m}$ and average connectivity strength within modules, $S$, and between modules, $W$. More precisely, we want to investigate if the response of the system is really scale-free, namely if avalanches of all sizes are observed, and to enlighten the role of modularity features.

Previous results ${ }^{19,22,25}$ have shown that the model generates a critical avalanche activity, i.e., an avalanche size distribution behaving as a power law over a size range up to the total number of neurons. This result is recovered on many non-modular complex network structures (regular, small world, scale free, fully connected). In order to investigate the role of modularity and, more precisely, of the module size on the avalanche activity, we first analyse a system where all modules have the same number of neurons $N_{m}$. On a modular network the avalanche size distribution exhibits a power law behavior only for avalanche sizes up to the size of a single module followed by an exponential decay (Fig. 2) and increasing $N_{m}$ leads to the occurrence of larger avalanches. The value of the power law exponent is found to be $1.7 \pm 0.11$ by a Kolmogorov-Smirnov test with a confidence level $90 \%$. This value is compatible but slightly larger than the experimental value for spontaneous activity ${ }^{9}$ and the numerical value found for this model on a non-modular network ${ }^{19,27}$. This could be due to the limited scaling regime which is of the order of the single module size.

Avalanches are not confined within the module where they start. Indeed, by direct inspection, we have verified that avalanches can reach several modules but are able to activate only a few neurons in the invaded modules. Avalanches are therefore able to carry information to different modules but the invasion of more modules results in a depression of the overall activity: Because of the weaker intermodule connections, activation of a single neuron in a new module leads to a limited activity in an environment not previously stimulated by this avalanche. Indeed, by rescaling the avalanche size by the number of neurons in a module $N_{m}$ (lower inset Fig. 2), we notice that the smaller the module size, the higher the probability to involve in the activity neurons in different modules. The dependence of the scaling regime on the number of modules is then analysed by increasing $M$, keeping $N_{m}$ fixed (upper inset Fig. 2). Interestingly, all distributions for different numbers of modules $M$ collapse onto a unique curve: The activity depression effect following the activation of more modules is solely controlled by the modular size $N_{m}$.

Experimental results ${ }^{5}$ indicate that intra-module connections are stronger than inter-module ones. We have verified if the average

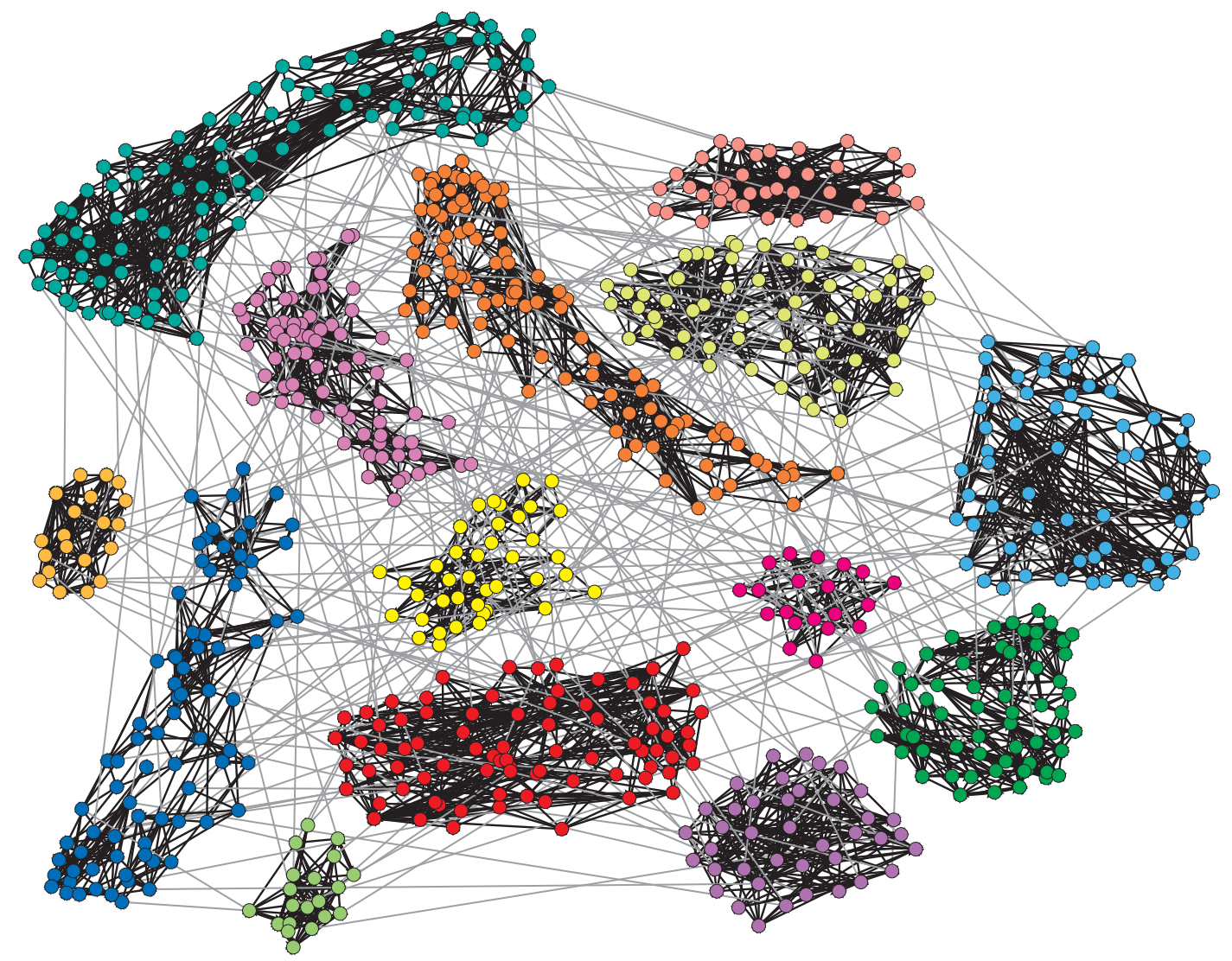

Figure $1 \mid$ Modular structure of the neuronal system. Network with $M=14$ modules in different colors with a number of neurons from $N_{m}=20$ to 100 . Intra-module connections have a larger average strength (thicker lines) than inter-module connections, i.e. $S>W$. For the network generation procedure see Methods. 




Figure $2 \mid$ Dependence of the scaling behaviour of the avalanche size distribution on system size. Avalanche size distribution for 100 configurations of modular networks with $M=25, W=0.2, S=0.4$ and for different values of $N_{m}$. The dashed line has a slope 1.7. Lower inset: The same curves plotted as function of $s / N_{m}$. Upper inset: Distributions evaluated for $N_{m}=900$ and different module numbers $M$.

connection strengths plays a role in limiting the scaling behavior of the avalanche distribution by changing $S$ and $W$, up to $W=S$ (Fig. 3). As expected, at fixed $N_{m}$ and $W$, larger avalanches become more probable for increasing $S$ (blue line and triangles). Conversely, by progressively increasing $W$ at fixed $S$ we observe that surprisingly the scaling regime decreases and the distributions extend to smaller avalanche sizes (curves with symbols). Indeed stronger inter-module connections make the invasion of different modules more probable, with a consequent depression of the overall activity as discussed above. Therefore, confining activity within a single module makes larger avalanches more probable. However, this effect becomes less relevant for increasing modular size $N_{m}$ and avalanches involving the entire system are never observed even for $W=S$, independently of their value (black circles and green line), suggesting that the only relevant parameter is the ratio $W / S$. For large $N_{m}$ the exponent value is independent of $S$ and $W$ and $W$ solely affects the onset of the exponential cut-off.



Figure $3 \mid$ Dependence of the scaling behaviour of the avalanche size distribution on synaptic strengths. Avalanche size distribution for 100 configurations of modular networks with $M=16, N_{m}=900$ and different values of $W, S$. The dashed line has a slope 1.7. Inset: Distributions for a smaller module size $N_{m}=200$.

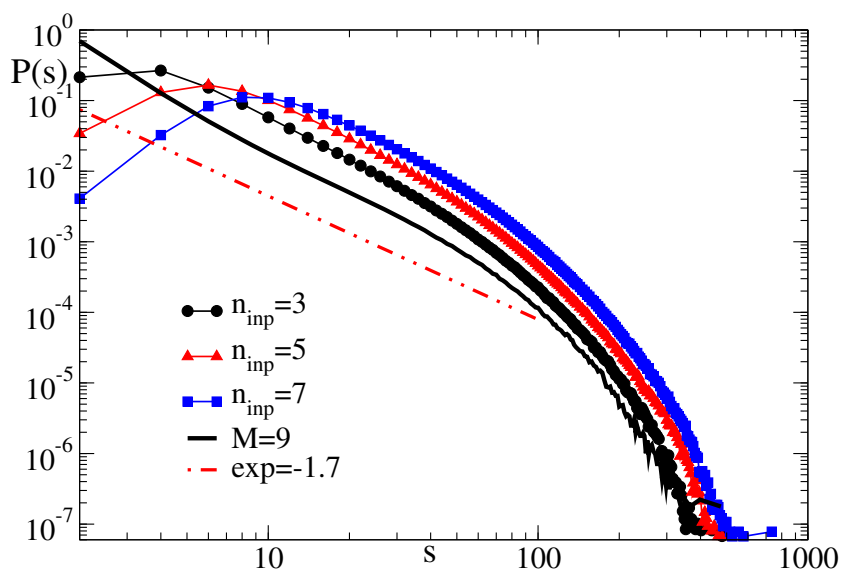

Figure 4 Scaling behaviour for multiple stimulation and different module size. Avalanche size distribution for 100 configurations of modular networks where $n_{i n p}$ neurons are initially stimulated. Parameter values are $M=16, N_{M}=400, W=S=0.4$ (symbols). Avalanche size distribution for networks with different $N_{m}\left(M=9, S=0.4, W=0.2, N_{m}\right.$ $=100,400,900)$. The dot-dashed line has a slope 1.7 .

In real brains external stimulation often involves more functional regions. We have monitored the system activity under the simultaneous stimulation of several neurons in different modules. By increasing the number of stimulated neurons chosen at random in different modules, the size of the largest avalanche increases, however activity is never able to involve all neurons even in the limiting case $W=S$ (Fig. 4). Conversely, under stimulation of several neurons a lack of small avalanches is observed, which further limits the scaling regime of the avalanche size distribution. As a final verification, we consider a network made of modules with different sizes (Fig. 4). The presence of small and large modules increases the occurrence probability of large avalanches, i.e., events involving about half system are observed. However, the scaling regime is again controlled by the size of the largest module. Interestingly, in all cases the value of the power law exponent is independent of the features of the modular network.

In order to better investigate the role of modularity on the avalanche activity, we monitor the number of modules $m$ involved in each avalanche and measure the distribution $P(m)$ (Fig. 5). This exhibits a power law behavior $P(m) \sim m^{-\mu}$ over a scaling range increasing with the total number of modules in the network $M$, followed by an exponential cut-off. In the upper inset we show that the scaling $P(m)=m^{-\mu} f\left(m / m_{\max }\right)$ is satisfied, with the maximum number, $m_{\text {max }}$, of modules reached by activity scaling with the number of modules in the network, $m_{\max } \propto M^{\sigma}$ with $\sigma=0.70 \pm 0.05$. As expected, for fixed $S$ the probability to invade more modules increases for larger $W$ and for $W \lesssim S$ avalanches reaching all modules can be observed. As a consequence the scaling behavior depends on $W$ for fixed $S$. More precisely, the power law exponent is solely dependent on the ratio $\beta=W / S$ (lower inset Fig. 5) and exhibits a value $\mu=3.6 \pm 0.1$ for $\beta>0.5$. The value of the exponents $\mu$ and $\sigma$ are obtained by a Kolmogorov-Smirnov test with a confidence level $95 \%$. The distribution crosses over towards an exponential behavior for smaller $\beta$. As observed in real brains, inter-module connections being slightly, but not significantly, weaker than connections within modules improve the functional efficiency of the system, since activity can reach all modules optimizing information transmission.

Results indicate that neither the module size, nor the number of modules, the stimulation extension or the connection strengths can make the modular network recover a truly critical behavior for avalanche activity. Modularity in the network is insured by the very different percentage of intra-module (92\%) and inter-module (8\%) connections. Increasing this last percentage would progressively modify and finally destroy the modular feature of the network. 


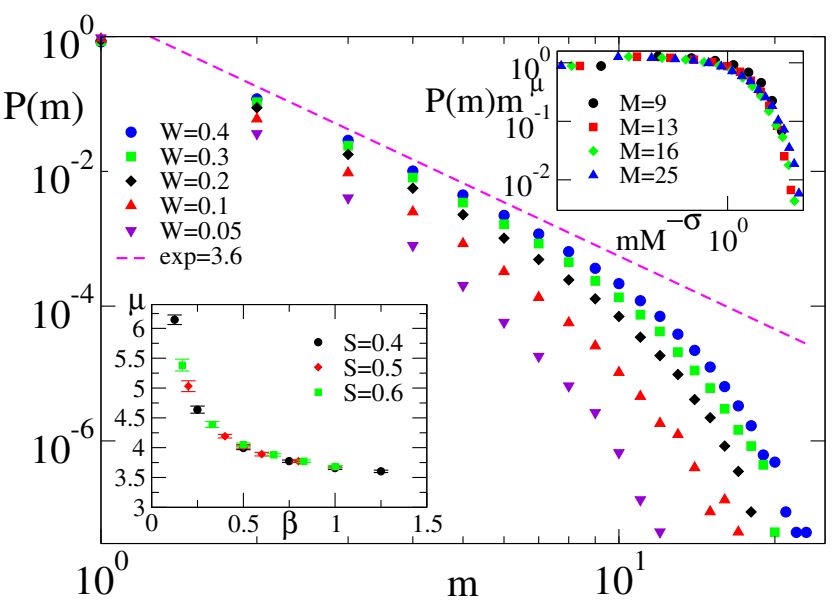

Figure 5 Scaling properties of the distribution of modules invaded by avalanches. Distribution of the number of modules reached by an avalanche for 100 configurations of modular networks with $M=25, N_{m}=$ 900 for different values of $W$ and fixed $S=0.4$. The dashed line indicates the power law decay with an exponent $\mu=3$.6. Lower inset: The power law exponent $\mu$ as function of $\beta=W / S$ for different $W$ and $S$. Upper inset: Universal scaling function obtained by rescaling of the distributions $P(m) m^{\mu}$ versus $m M^{-\sigma}$.

Indeed, by increasing the number of inter-module connections the avalanche size distribution finally recovers a power law behavior over the entire scaling range (Fig. 6) if connections are equally distributed within and between modules. This effect is observed even more clearly for longer plastic adaptation, namely for a wider distribution of synaptic strengths (red straight line). Conversely, for modular networks ( $8 \%$ of inter-module connections) the distribution is not affected by the synaptic strengths and the two distributions for different connection strengths collapse (black symbols and grey straight line). Moreover, for increasing inter-module connections avalanches have a higher probability to invade more modules (inset of Fig. 6) and for $50 \%$ inter-module connections avalanches invade with equal probability all modules.

\section{Discussion}

Modular organization is a characteristic feature of many biological systems, especially relevant in systems exhibiting separate functional units. In neuronal networks, modularity plays a crucial role in network synchronization and affects the synchronization transition more than long-range connections ${ }^{28}$. Hierarchical modular structure has been also found able to sustain scale-free activity in presence of weak perturbations ${ }^{23}$. However, how the modular structure affects the scale free behaviour is an open question which can provide interesting insights in the origin of pathological response in some neuronal systems. The present study shows evidence that the modular structure of the brain, made of highly connected functional areas loosely inter-connected by weaker links, is a fundamental ingredient insuring efficient and controlled functioning of healthy brains. Modularity, at the same time, allows information to reach all areas but hinders the involvement of the whole system in activity, which could lead to pathological response. This behaviour is due to an activity depression resulting from the invasion through weaker links of modules not stimulated by previous firings, which tend to confine the response to the modular size. These results are in agreement with experimental data, since the critical behavior of brain activity is confirmed at the level of single functional areas, i.e., non-modular networks with different connectivity properties. At the scale of the entire brain, conversely, our results explain why critical behaviour should not be observed, regardless the modular size, the number of modules and the connectivity strengths. The only way to recover

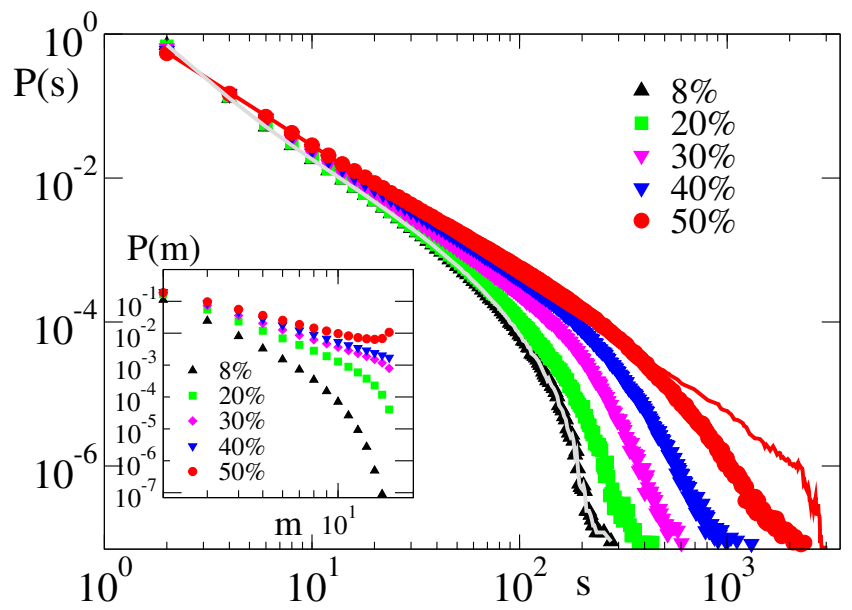

Figure 6 Destruction of modularity leads to recovery of full scale free behaviour. Avalanche size distribution for 100 configurations of networks with $M=16$ and $N_{m}=200$ neurons and for increasing percentage of intermodule connections ( $W=S=0.6$ ) and limited plasticity (symbols). Straight lines show the same distributions for $8 \%$ and $50 \%$ and longer plastic adaptation (grey and red lines respectively). Inset: Distributions $P(m)$ for the same networks.

criticality is to increase inter-modular connectivity, transforming the modular network and leading to the loss of modularity of the functional network, which becomes a more random structure, as in patients with neurological diseases. This suggests that the structure could be at the origin of such pathologies. Results are in agreement with a study of the stability of the power grid in US via the sand-pile model on a two-module network ${ }^{29}$. Also in this case modularity mitigates large cascades by diverting load at an optimal percentage of inter-module connections close to $8 \%$.

\section{Methods}

Implementation of the modular network. Here we implement a single level of the modular network measured in ref. 5 (Fig. 1). We consider $N$ neurons uniformly distributed in a $2 d$ system. Neurons are organized in $M$ modules each containing $N_{m}$ neurons. The simulated values of $M$ are comparable with the number of functional areas or Brodmann's area in the brain, under the requirement that $N_{m}$ is large enough to provide a satisfactory scaling regime for the size distributions. Both, the case of modules with the same and different size are analysed. Synaptic connections and strengths $g_{i j}$ are assigned according to ref. 5, with $g_{i j} \neq g_{j i}$. More precisely, connections between neurons within the same module form a scale-free network, where the outgoing connectivity degree is distributed as $n\left(k_{\text {out }}\right) \propto k_{\text {out }}^{-\gamma}$ with $\gamma=2.1$, and their initial value $g_{i j}$ is assigned at random in the interval $[S-0.1, S+0.1]$. The two neurons are chosen according to a distance dependent probability, $p(r) \propto e^{-r / 5<r>}$, where $r$ is their spatial distance and $\langle r\rangle$ a characteristic distance ${ }^{30}$. Results do not depend on the neuronal spatial density or the average connection length $\langle r\rangle$. Since the synaptic connections between modules represent $8 \%$ of the total number of connections ${ }^{5}$, we evaluate their number on the basis of the total number of intra-module connections as $N k_{\text {inter }}=N k_{\text {intra }} 0.08 / 0.92$. We then assign them according to

$P\left(k_{\text {out }}^{i}, k_{\text {out }}^{j}\right) \sim\left(k_{\text {out }}^{i}\right)^{1-\gamma}\left(k_{\text {out }}^{j}\right)^{-\epsilon}$, where $k_{\text {out }}^{i}$ and $k_{\text {out }}^{j}$ are the number of synapses of neurons $i$ and $j$ belonging to different modules and $\epsilon=2.1$, according to the experimental value. This implies that inter-module connections are typically established between low connectivity neurons and hubs are connected with neurons within the same module. Each inter-module connection has an initial random strength $g_{i j}$ in $[W-0.1, W+0.1]$, where $W<S$ according to ref. 5 . The total outconnectivity degree distribution is updated during each step of the procedure, to include both inter-module and intra-module connections. Once the network of output connections is established, we identify the resulting degree of in-connections, $k_{i n}$ for each neuron.

Neuronal network model. We consider $N$ neurons characterized by their potential $v_{i}$, initially assigned at random. The initial distribution is irrelevant in order to obtain critical avalanche activity. Each neuron can be excitatory or inhibitory, with a percentage of $p_{i n}=10 \%$ inhibitory synapses. This value, lower than the percentage measured experimentally, is needed in order to observe a sufficient scaling regime in avalanche size distributions. As it will be shown in the Results section, the scaling regime is controlled by the single module rather than the entire system size, therefore a larger $p_{i n}$ would require to simulate networks with larger $N_{m}$. Moreover, inhibitory 
neurons are chosen among neurons with high connectivity $\left(k_{\text {out }}>50\right)$, according to experimental observations ${ }^{31}$. Each neuron fires as soon as its potential reaches a threshold $v_{\max }$. In order to start activity a small random stimulus (about $v_{\max } / 10$ ) is applied to a random neuron. As soon as at a given time the value of the potential at a neuron $i$ is above threshold, $v_{i} \geq v_{\max }$, the neuron fires modifying the potential of the $k_{\text {out }}$ connected neurons proportionally to $g_{i j}{ }^{27}, v_{j}(t+1)=v_{j}(t) \pm \frac{v_{i} k_{\text {out }}^{i}}{k_{\text {in }}^{j}} \frac{g_{i j}(t)}{\sum_{k} g_{i k}(t)}$, where the sum over $k$ is over all out-going connections of $i$ and the plus or minus sign denotes excitatory or inhibitory $g_{i j}$, respectively. The ratio $k_{\text {out }}^{i} / k_{i n}^{j}$ makes the potential variation of neuron $j$ induced by neuron $i$ independent of the connectivity level of both neurons, as expected for real neurons ${ }^{27}$. After firing, a neuron is set to a zero resting potential and in a refractory state lasting one time step (about $10 \mathrm{~ms}$ ), during which it is unable to receive or transmit any charge. At the end of an avalanche, we implement Hebbian plasticity rules: The strength of synapses connecting active neurons is increased proportionally to the activity of the synapse, i.e., the potential variation of the post-synaptic neuron, $g_{i j}(t+1)=g_{i j}(t)+\left(v_{j}(t+1)-v_{j}(t)\right) / v_{\max }$ Conversely, the strength of all inactive synapses is reduced by the average strength increase per synapse, $\Delta g=\sum_{i j, t} \delta g_{i j}(t) / N_{b}$, where $N_{b}$ is the number of synapses. The same strengthening and weakening rules are applied to both excitatory and inhibitory synapses. The presence of both strengthening and weakening rules implements a homeostatic regulatory mechanism for synaptic strengths, which preserves the average synaptic strength ${ }^{32}$. An external stimulus then triggers further activity in the system. We implement plasticity during a finite series of stimuli in order to modify the synaptic strengths initially assigned at random. However, we verify that plastic adaptation preserves the average values of the strength distribution, in particular that inter-module connections are weaker than intra-module ones. Moreover, we limit the duration of plastic adaptation to avoid pruning of synaptic strengths, which would modify the network modular structure.

1. Eguiluz, V. M., Chialvo, D. R., Cecchi, G. A., Baliki, M. \& Apkarian, A. V. Scalefree brain functional networks. Phys. Rev. Lett. 94, 018102 (2005).

2. Sporns, O. \& Zwi, J. D. The small word of the cerebral cortex. Neuroinformatics 2 , 145-162 (2004).

3. Rubinov, M. et al. Small-world properties of nonlinear brain activity in schizophrenia. Human Brain Mapping 30, 403-416 (2009).

4. Bartolomei, F. et al. Disturbed functional connectivity in brain tumour patients: evaluation by graph analysis of synchronization matrices. Clinical Neurophysiology 117, 2039-2049 (2006).

5. Gallos, L. K., Makse, H. A. \& Sigman, M. A small-world of weak ties provides optimal global integration of self-similar modules in functional brain networks. Proc. Natl. Acad. Sci. USA 109, 2825-2830 (2012).

6. Kleinberg, J. M. Navigation in a small world. Nature 406, 845 (2000).

7. Li, G. et al. Towards Design Principles for Optimal Transport Networks. Phys. Rev. Lett. 104, 018701 (2010).

8. Li, G. et al. Optimal transport exponent in spatially embedded networks. Phys. Rev. E 87, 042810 (2013).

9. Beggs, J. M. \& Plenz, D. Neuronal avalanches in neocortical circuits. J. Neurosci. 23, 11167-11177 (2003).

10. Beggs, J. M. \& Plenz, D. Neuronal avalanches are diverse and precise activity patterns that are stable for many hours in cortical slice cultures. J. Neurosci. 24, 5216-5229 (2004)

11. Gireesh, E. D. \& Plenz, D. Neuronal avalanches organize as nested theta- and beta/ gamma-oscillations during development of cortical layer 2/3. Proc. Nat. Acad. Sci. USA 105, 7576-7581 (2008).

12. Petermann, T. et al. Spontaneous cortical activity in awake monkeys composed of neuronal avalanches. Proc. Nat. Acad. Sci. USA 106, 15921-15926 (2009).

13. Mazzoni, A. et al. On the dynamics of the spontaneous activity in neuronal networks. PLoS ONE 2, e439 (2007).

14. Pasquale, V., Massobrio, P., Bologna, L. L., Chiappalone, M. \& Martinoia, S. Selforganization and neuronal avalanches in networks of dissociated cortical neurons. Neuroscience 153, 1354-1369 (2008).
15. Fraiman, D. \& Chialvo, D. R. What kind of noise is brain noise: anomalous scaling behavior of the resting brain activity fluctuation. Front. Physiol. 3, 307 p1-9 (2012).

16. Shriki, O. et al. Neuronal avalanches in the resting MEG of the human brain. J. Neurosci. 33, 7079-7090 (2013).

17. Zapperi, S., Lauritsen, K. B. \& Stanley, H. E. Self-organized branching processes: mean-field theory for avalanches. Phys. Rev. Lett. 75, 4071-4074 (1995).

18. Lauritsen, K. B., Zapperi, S. \& Stanley, H. E. Self-organized branching processes: avalanche models with dissipation. Phys. Rev. E 54, 2483-2488 (1996).

19. de Arcangelis, L., Perrone Capano, C. \& Herrmann, H. J. Self-organized criticality model for brain plasticity. Phys. Rev. Lett. 96, 028107 (2006).

20. Levina, A., Herrmann, J. M. \& Geisel, T. Dynamical synapses causing selforganized criticality in neural networks. Nature Phys. 3, 857-860 (2007).

21. Millman, D., Mihalas, S., Kirkwood, A. \& Niebur, E. Self-organized criticality occurs in non-conservative neuronal networks during 'up' states. Nature Phys. 6, 801-805 (2010)

22. de Arcangelis, L. \& Herrmann, H. J. Activity-dependent neuronal model on complex networks. Front. Physiol. 3, 62 p1-9 (2012).

23. Wang, S. J., Hilgetag, C. C. \& Zhou, C. Sustained activity in hierarchical modular neural networks: self-organized criticality and oscillations. Front. Comp. Neurosci. 5, 30 p1-13 (2011).

24. Stoop, R. et al. Beyond scale-free small-world networks: cortical columns for quick brains. Phys. Rev. Lett. 110, 108105 (2013).

25. Pellegrini, G. L., de Arcangelis, L., Herrmann, H. J. \& Perrone Capano, C. Modelling the brain as an Apollonian network. Physical Review E 76, 016107 (2007).

26. de Arcangelis, L. \& Herrmann, H. J. Learning as a phenomenon occurring in a critical state. Proc. Natl. Acad. Sci. USA 107, 3977-3981 (2010).

27. Lombardi, F., Herrmann, H. J., Perrone-Capano, C., Plenz, D. \& de Arcangelis, L. Balance between excitation and inhibition controls the temporal organization of neuronal avalanches. Phys. Rev. Lett. 108, 228703 (2012).

28. Fuchs, E., Ayali, A., Ben-Jacob, E. \& Boccaletti, S. The formation of synchronization cliques during the development of modular neural networks. Phys. Biol. 6, 036018 (2009).

29. Brummitt, C. D., D’Souza, R. M. \& Leicht, E. A. Suppressing cascades of load in interdependent networks. Proc. Natl. Acad. Sci. USA 109, E680 (2012).

30. Roerig, B. \& Chen, B. Relationships of local inhibitory and excitatory circuits to orientation preference maps in ferret visual cortex. Cerebral Cortex 12, 187-198 (2002).

31. Bonifazi, P. et al. GABAergic hub neurons orchestrate synchrony in developing hippocampal networks. Science 326, 1419-1424 (2009).

32. Royer, S. \& Paré, D. Conservation of total synaptic weight through balanced synaptic depression and potentiation. Nature 422, 518-522 (2003)

\section{Acknowledgments}

We acknowledge financial support from the ERC Advanced Grant 319968-FlowCCS and the SNF project 205321-13874.

\section{Author contributions}

L.d.A. and H.J.H. were involved in all the phases of this study. R.R. performed the numerical simulations and prepared the figures. All authors reviewed the manuscript.

\section{Additional information}

Competing financial interests: The authors declare no competing financial interests.

How to cite this article: Russo, R., Herrmann, H.J. \& de Arcangelis, L. Brain modularity controls the critical behavior of spontaneous activity. Sci. Rep. 4, 4312; DOI:10.1038/ srep04312 (2014)

(c) (i) $\Theta$ This work is licensed under a Creative Commons Attributioncc. visit http://creativecommons.org/licenses/by-nc-nd/3.0 